\title{
Evaluation of Protocols of Controlled Ovarian Stimulation in Obtaining Mature Oocytes (MII): Retrospective Study on Assisted Reproductive Technology Procedures
}

\author{
Cátia Manuela Ribeiro Barbosa Martins ${ }^{1}$, Patrícia Cordeiro Pires de Figueiredo Gomes Crisóstomo Ruivo ${ }^{1,2}$, \\ Denise Cristina Mós Vaz-Oliani ${ }^{1,2,3}$, Renato Alessandre Silva Martins ${ }^{1,2}$, Antonio Helio Oliani ${ }^{1,2,3}$ \\ ${ }^{1}$ University of Beira Interior - Faculty of Health Sciences, Covilhã, Portugal \\ ${ }^{2}$ Reproductive Medicine Unit - University Hospital Center Cova da Beira, Covilhã, Portugal \\ ${ }^{3}$ Institute of Reproductive and Fetal Medicine - Faculty of Medicine of São José do Rio Preto, São José do Rio \\ Preto, Brazil
}

This study was presented at the:

University of Beira Interior - Faculty of Health Sciences to obtain the Master's Degree in Medicine (Integrated Master)

\begin{abstract}
Objective: To understand which of the controlled ovarian stimulation (COS) protocols used in different patients are associated with greater amounts of oocytes retrieved.

Methods: The study population was divided into three groups, considering AMH and AFC to obtain the Ovarian Response Predictor Index (ORPI); they were grouped into: G1-Low Reserve (ORPI <0.5); G2-Normal Reserve (ORPI:0.5-0.9); and G3-High Reserve (ORPI $\geq 0.9$ ). 246 cycles were selected in which COS was used: recombinant FSH - follitropin alfa or beta (Protocol 1) or corifollitropin alfa (Protocol 2), both associated with urinary HMG and the GnRH antagonist, with the trigger performed using recombinant hCG or GnRH agonist.

Results: The number of oocytes obtained was higher in protocol 1 in all groups, with higher counts seen in G1 than in G2 or G3. The number of days required in COS for protocol 2 was greater than for protocol 1 in all groups. The total dose of recombinant FSH alfa or beta / urinary HMG used in protocol 1 was inversely proportional to the ovarian reserve. The lower the ORPI, the greater the average number of international units administered. In protocol 2 , there was a need to supplement with higher doses of urinary HMG when compared to protocol 1 . The dosage of the GnRH antagonist was dependent on the number of COS days until the trigger was used. In obtaining MII oocytes, the percentages were similar regardless of the trigger used.

Conclusions: The use of follitropin leads to greater numbers of retrieved oocytes than corifollitropin alfa in all ORPIs. The dose of recombinant FSH used with urinary HMG increases inversely proportional to the ORPI value. The fixed dose of recombinant FSH deposit requires a sharp increase in the dose of urinary HMG.
\end{abstract}

Keywords: infertility, assisted reproductive technology, anti-Müllerian hormone, antral follicle count, controlled ovarian stimulation

\section{INTRODUCTION}

The World Health Organization (WHO) has considered infertility a Public Health problem. It has been defined as "a disease of the reproductive system translated into the inability to obtain a pregnancy after 12 months or more of regular sexual intercourse and without using contraception" (Direcção Geral de Saúde, 2010; Vander Borght \& Wyns, 2018).
The prevalence of infertility is exceedingly difficult to assess, with studies reporting the possibility that one in four women may have difficulties in becoming pregnant throughout their lives (Direcção Geral de Saúde, 2010). Recent studies report a prevalence of infertility that varies between $3.5 \%$ and $16.7 \%$ in the most developed countries and $6.9 \%$ to $9.3 \%$ in the least developed countries (Direcção Geral de Saúde, 2010; Vander Borght \& Wyns, 2018)

In view of these data, and since the prevalence of infertility has grown globally, couples are more actively seeking reproductive health clinics (Gnoth et al., 2005). In Portugal, there has been an increase in activity in this area. However, this increase is still insufficient to respond to the problems of couples with indication for Assisted Reproductive Technology (ART) treatments.

Evidence suggests that the prevalence of infertility continues to increase. The causes for such increase include the decision by couples of having children at a later age, increased obesity, consumption of tobacco, alcohol, and other harmful substances, and the increasing exposure of couples to high levels of stress. (Direcção Geral de Saúde, 2010; Vander Borght \& Wyns, 2018; Deshpande \& Gupta, 2019)

The relationship between a woman's age and fertility is already well known; the older the age, the lower the quantity and quality of oocytes (Vollenhoven \& Hunt, 2018; Liu \& Case, 2011). At birth, the human ovary contains one to two million primordial quiescent follicles. Only a small part of these (between 300 and 400,000 ) will mature during a woman's reproductive life. Nuclear maturation of oocytes, accompanied by cytoplasmic maturation, shows their evolution from the prophase I stage, during recruitment and follicular development, to metaphase II (stage MII), which occurs in final maturation and subsequent ovulation (Vollenhoven \& Hunt, 2018; Liu \& Case, 2011; Braga et al., 2020).

The ovarian reserve describes the functional potential of the ovary, reflecting the quantity and possible quality of oocytes. The antral follicles count (AFC) is defined as the number of follicles with a diameter of less than $10 \mathrm{~mm}$ evaluated in the early follicular phase. It is a good marker of ovarian reserve obtained via endovaginal ultrasound examination of the ovaries (Silva \& Vilodre, 2009; Amanvermez \& Tosun, 2016).

The anti-Müllerian hormone (AMH) is secreted by the granulosa cells of the pre-antral and growing antral follicles. AMH production occurs during follicle differentiation from the primordial to the primary phase and continues until the follicles reach stages with diameters from 2 to $6 \mathrm{~mm}$. 
Some authors describe AMH values as low when they are < $0.5 \mathrm{ng} / \mathrm{mL}$; intermediate when they range between 1.0 and $3.5 \mathrm{ng} / \mathrm{mL}$; and high when they are $>3.5 \mathrm{ng} / \mathrm{mL}$. Although it is important to estimate the ovarian reserve, AMH cannot be used to assess oocyte or embryo quality. Compared to AFC, AMH has the advantage of showing little variation over the menstrual cycle and between cycles. (Dewailly \& Laven, 2019; Usta \& Oral, 2012; La Marca et al., 2010).

AFC, AMH, and female age are important parameters to predict response to controlled ovarian stimulation (COS) and identify women who are unresponsive to treatment, at risk of canceling the cycle, and hyper-responsive women at higher risk of developing Ovarian Hyperstimulation Syndrome (OHSS) (Oliani et al., 2019).

The Ovarian Response Prediction Index (ORPI) is calculated using the formula below:

\section{$\mathrm{ORPI}=(\mathrm{AMHxAFC}) /$ Woman age}

The ORPI may aid in the choice of type of COS and help predict patient response to the proposed treatment based to her index, as follows: poor response is expected in women with an ORPI <0.5; women with an ORPI of [0.5-0.9] are expected to present optimal response to treatment; and women with an ORPI $\geq 0.9$ are expected to present hyper-response to treatment (Oliani et al., 2019; Oliveira et al., 2012). With COS, the goal is to obtain an adequate number of quality oocytes for each treatment cycle.

In vivo, oocyte maturation occurs because of natural selection. In contrast, in ART cycles, growth and maturation of multiple follicles is induced using supra-physiological doses of gonadotropins, to make sure a desired number of mature oocytes is obtained (Braga et al., 2020; Hodgen, 1989; Quaas \& Legro, 2019). Different COS protocols entail different combinations of hormones that may lead to follicular asynchrony and variations in the quantity, quality, viability, and competence of oocytes. Therefore, it is extremely important to analyze the quantity and quality of stimulation given to patients (Braga et al., 2020; Quaas \& Legro, 2019)

It is important that patients undergo preparation before the initiation of COS. To facilitate scheduling for women undergoing treatment and ART centers, combined oral contraceptives (COC) have been prescribed prior to the initiation of treatment cycles to schedule and date menstruation. According to studies (Biljan et al., 1998; Griesinger et al., 2008), the use of COC provides other advantages, such as improved follicular timing during ovulation stimulation, lower cancellation rates in hyper-responsive women, and greater number of aspirated oocytes.

Gonadotropins are essential in COS protocols. Follicle-Stimulating Hormone (FSH) is the main hormone in ovulation induction. Various preparations contain FSH and can be used in ovulation stimulation cycles for ART (Oliani et al., 2019). The three most used forms are: (1) Urinary Human Menopausal Gonadotropin (UHMG), which contains FSH and Luteinizing Hormone (LH) of urinary origin and is extracted from the urine of menopausal women (Deeks, 2018); (2) highly purified urinary FSH, which contains predominantly urinary FSH (uFSH) and is also extracted from the urine of menopausal women, although it undergoes a process of greater purification from which urinary $\mathrm{LH}$ (uLH) (Oliani et al., 2019) and Recombinant FSH (rFSH) are extracted, the latter of which contains only FSH (alfa or beta follitropins); it is produced in a laboratory setting and offers unlimited production potential, unlike UHMG and uFSH. Currently, there is also a recombinant form of prolonged release $\mathrm{FSH}$, corifollitropin alfa (Oliani et al., 2019; Rombauts \& Talmor, 2012).
Corifollitropin alfa is a recombinant gonadotropin with pharmacodynamic properties similar to conventional rFSH, but with distinct pharmacokinetic characteristics conferring it clinically relevant practical advantages. A single injection of corifollitropin alfa can initiate and maintain multiple follicular developments during the first seven days of ovarian stimulation prior to Ovum Pick Up (OPU) and In Vitro Fertilization (IVF) or Intracytoplasmic Sperm Injection (ICSI), thus requiring fewer drug interventions (Ledger et al., 2011). In contrast with the use of daily FSH preparations, dose adjustments with corifollitropin alfa are not possible during the first seven days of ovarian stimulation, since corifollitropin alfa remains pharmacologically active during this period (Ledger et al., 2011).

In ART cycles, Gonadotropin-Releasing Hormone $(\mathrm{GnRH})$ analogs are also used. They are not ovulation inducers, but endocrine modulators used during ovulation stimulation to avoid the spontaneous early peak of LH, responsible for ovulation and follicular luteinization (Oliani et al., 2019). Natural GnRH is a protein, produced by the hypothalamus, with pulsatile release, which has good affinity for its pituitary receptor and a short half-life. GnRH stimulates the pituitary to release FSH and LH. The synthesized GnRH analogs (antagonists and agonists) have a longer half-life than natural $\mathrm{GnRH}$ and a greater affinity for pituitary receptors (Oliani et al., 2019; Newton et al., 2018). Antagonists act by competitive inhibition with natural GnRH in the occupation of receptors, thus creating an immediate pituitary block, with effects observed within a few hours, suppressing the release of FSH and LH. Suppression is more important for LH than for FSH (Oliani et al., 2019; Newton et al., 2018). GnRH agonists rapidly stimulate the pituitary to release $\mathrm{FSH}$ and $\mathrm{LH}$. With the continuous occupation of the same receptors, there is a progressive desensitization over 14 days, with consequent initial elevation and subsequent suppression of FSH and $\mathrm{LH}$ secretion (Oliani et al., 2019; Newton et al., 2018).

$\mathrm{GnRH}$ analogues prevent a premature LH surge and avoid the loss of the cycle resulting from early ovulation. Several studies have shown that GnRH antagonists are more potent suppressors than agonists (Oliani et al., 2019). The LH peak at the end of folliculogenesis plays an important role in activating oocyte meiosis, with the release, after 36 hours, of a mature oocyte (MII) capable of being fertilized. In COS cycles, when the pituitary is blocked (by $\mathrm{GnRH}$ agonists or antagonists), there is no spontaneous LH peak; therefore, the peak must be stimulated using a trigger. The ideal time to use the trigger is when follicles between 18 and $20 \mathrm{~mm}$ are viewed in the ultrasound examination performed during COS (Oliani et al., 2019).

Human Chorionic Gonadotropin (hCG) can be used as a trigger; in cases in which an antagonist is used in pituitary block, a GnRH agonist should be chosen (Alyasin et al., 2016; Le et al., 2019). hCG is a gonadotrophic glycoprotein hormone, produced mainly by placental trophoblasts, as the LH produced by the pituitary gland and used in the final maturation of oocytes. hCG can be of urinary origin (uhCG) or recombinant (rhCG), the latter being the one the most used in COS (Youssef et al., 2016). GnRH agonists, in addition to being used to block the hypothalamic-pituitary axis, are also used as a trigger for the endogenous release of $\mathrm{LH}$ and for the final maturation of oocytes, in cycles in which a GnRH antagonist was used and to prevent complications of ovarian hyperstimulation (Dosouto et al., 2017).

Patients with OHSS, a condition currently considered as an iatrogenic syndrome, have multiple follicles and develop ascites, hematological changes, pleural effusion, liver or coagulation disorders which can, in rare cases, be severe or even fatal (Blumenfeld, 2018) OHSS stems from 
excessive response to hormones used in ovarian stimulation. It is the most feared complication in ART, due to its severity. In the past decade, the replacement of hCG by $\mathrm{GnRH}$ agonists in women at risk of hyperstimulation has decreased the incidence and severity of OHSS (Blumenfeld, 2018). The use of GnRH agonists to trigger ovulation is the best option to prevent OHSS and maintain acceptable reproductive results (Blumenfeld, 2018).

The several hormones currently available for COS indicate that there has been a continuous search for a protocol that ofers the best hormonal combination and provides good follicular response in the safest, most efficient, and physiological way possible. However, it has not yet been possible to conclude which is the best protocol.

\section{Study Objectives}

The objective of this study was to understand which of the COS protocols used in different patients was associated with greater numbers of oocytes retrieved during OPU, taking into account the number of days of stimulation, the doses of hormones administered, and which trigger obtained greater numbers of mature oocytes (stage MII) for fertilization. Other factors considered were patient age, AFC, and AMH levels, since they are correlated with ORPI and may directly or indirectly influence the success of ART treatments.

Based on the objectives of this study, the following main hypotheses were formulated: there is a significant difference between protocols in the number of oocytes obtained; there is a significant difference between protocols in the length of stimulation; there is a significant difference between protocols in the required stimulation dose; and there is a significant difference between triggers in the percentage of mature oocytes obtained.

\section{MATERIALS AND METHODS}

\section{Type of study}

This retrospective observational study was carried out based on the analysis of clinical processes of patients submitted to ART (exclusively IVF and ICSI) at the Reproductive Medicine Unit of the Hospital and University Center of Cova da Beira (CHUCB) between January 1, 2015 and December 31, 2019.

\section{Data collection}

The data of the patients included in this study were obtained from the clinical files saved in the archives of the Reproductive Medicine Unit at CHUCB. Data collection was carried out in September 2020, after a favorable opinion from the Health Ethics Committee. The data collected from each process were patient age, AFC, AMH level, drugs used in COS, stimulation dosage and length, used trigger, total number of oocytes obtained, and number of mature oocytes only. The data were collected without alterations by the researcher.

Study participants, inclusion and exclusion criteria, and sample matching

The study population included female patients who underwent IVF or ICSI at the Reproductive Medicine Unit of CHUCB between January 1, 2015 and December 31, 2019. A total of 212 women and 317 COS cycles were included. Of the 317 COS cycles performed in the five years of the study, we selected the cycles in which one of the following protocols were used:

Protocol 1 (203 cycles): rFSH alfa and beta (Gonal ${ }^{\circledR} /$

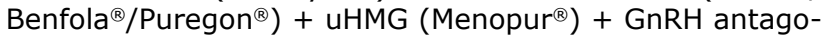
nist (Orgalutran ${ }^{\circledR} /$ Cetrotide $^{\circledR}$ ); and Protocol 2 (43 cycles): rFSH corifollitropin alfa (Elonva $\left.{ }^{\circledR}\right)+$ uHMG $\left(\right.$ Menopur $\left.^{\circledR}\right)+$ GnRH antagonist (Orgalutran ${ }^{\circledR} /$ Cetrotide $^{\circledR}$ ). We decided to select these protocols because they amounted to a representative sample. A total of 246 COS cycles performed in 179 women were included; 124 women were submitted to only one cos cycle, 45 were submitted to two cycles, and 10 underwent three cycles. Female patient age varied between 19 and 39 years, with an average age of 34.51 years. The study included women with all the data required for the study of ovarian reserve, namely: Age, Anti-Müllerian Hormone level, and Antral Follicles count; individuals prescribed COS Protocol 1 or 2 and subjects undergoing IVF or ICSI. Patients with missing information for purposes of this study, individuals on regimans other than Protocols 1 and 2 described above, and women submitted to IVF or ICSI with previously frozen oocytes were excluded. For sample matching, the COS cycles were divided into three groups (G1, G2 and G3) according to ORPI and consequent Ovarian Reserve. G1 included subjects with an ORPI $<0.5$, rated as having low ovarian reserve; $\mathrm{G} 2$ included subjects with an ORPI of [0.5-0.9], rated as having normal ovarian reserve; and G3 included women with an ORPI $\geq 0.9$, rated as having a high ovarian reserve.

\section{Statistical methods and procedures}

IBM $^{\circledR}$ SPSS Statistics $21^{\circledR}$ (Statistical Package for the Social Sciences, Inc., Chicago, IL) was used for data treatment and analysis. The frequencies (absolute and relative) of categorical variables and descriptive statistics of continuous variables were analyzed. In order to compare the number of oocytes obtained and the number of days of stimulation (dependent variables) with the Protocol (independent variable or factor), and to compare the proportion of mature oocytes (dependent variable) with the trigger (independent variable), we used the parametric Student's t-test for independent samples. Student's t-test tests the null hypothesis that the means of the two groups are equal, against the alternative hypothesis that the means are different. A significant result ( $p$-value $<a)$ leads to the rejection of the null hypothesis of equality and allows the inference of significant differences between the two samples. The assumption of data normality was validated (required for the application of parametric tests): the hypothesis of normality of the continuous variable in groups of less than 30 was not rejected, according to the Shapiro-Wilk test ( $p$-value >0.05), and the shape of the approximate normal distribution in samples larger than 30 validated the application of Student's t-test. In addition, in samples of sufficiently large size (greater than 30) the Student's t-test is robust for small deviations from normality and the data distribution tends to Normal (by the Central Limit Theorem). A significance level of $5 \%$ $(a=0.05)$ was considered.

\section{RESULTS}

The COS cycles were stratified $(n=246)$ according to the ORPI values of women submitted to ART. Almost half $(48.4 \%)$ of the cycles $(n=119)$ were performed in individuals in G1, rated as having low ovarian reserve and an ORPI $<0.5 ; 46$ cycles $(18.7 \%)$ were perfomed in individuals in G2, rated as having normal ovarian reserve and an ORPI of [0.5-0.9]; and 81 cycles (32.9\%) were performed in women in G3, rated as having high ovarian reserve and an ORPI $\geq 0.9$.

Protocol 1 with $\mathrm{rFSH}$ alfa and beta (Gonal ${ }^{\circledR} /$ Benfola $^{\circledR} /$ Puregon ${ }^{\circledR}$ ) was prescribed in the majority of the COS cycles $(82.5 \%, n=203)$, while the remaining $17.5 \%(n=43)$ were offered Protocol 2 with rFSH corifollitropin alfa (Elonva ${ }^{\circledR}$ ). Patients on both protocols were prescribed uHMG (Menopur $^{\circledR}$ ) and offered rotection against early LH surge with a GnRH antagonist (Orgalutran ${ }^{\circledR} /$ Cetrotide $^{\circledR}$ ). Ovulation was triggered with rhCG (Ovitrelle ${ }^{\circledR}$ ) in $87.8 \%$ of the cycles 
$(n=216)$ and with a GnRH agonist (Decapepty ${ }^{\circledR} /$ Gonapeptyl ${ }^{\circledR}$ ) in $11 \%$ of the cycles.

Protocol 1 was used in 68.1\% $(n=81)$ and Protocol 2 in $31.9 \%(n=38)$ of the women with low ovarian reserve (ORPI <5) assigned to $\mathrm{G} 1$. The average number of oocytes obtained was significantly higher in Protocol 1

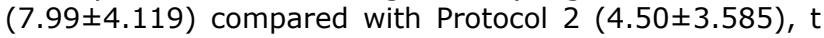
$(117)=4.228$ and $p$-value $<0.01$; therefore, it is possible to reject the null hypothesis of equality of means between the two protocols. There is thus statistical evidence that the average number of oocytes obtained differs between the protocols, with an average difference of about three oocytes, with patients on Protocol 1 having, on average, three more oocytes than subjects on Protocol 2. The mean number of stimulation days was significantly higher in Protocol $2(11.39 \pm 1.264)$ than in Protocol $1(10.80 \pm 1.123), t$ $(117)=-2.576$ and $p$-value $<0.05$ with an average difference of 0.592 days. There is thus statistical evidence that the average number of stimulation days differs significantly between the two protocols.

The following boxplots (diagrams of extremes and quartiles) represent the distribution of data for each protocol regarding the number of oocytes obtained and the number of stimulation days. Figure 1 shows a greater variability in the number of oocytes obtained in Protocol 1 ; in Protocol 2, a smaller range of values is observed, and the median is smaller (horizontal line in the center of the box). The median number of oocytes in Protocol 1 was 7, while $50 \%$ of Protocol 2 patients had up to 4 oocytes (Median = $4)$. There are two outliers in Protocol 1 and one outlier in Protocol 2. Outliers are values that exceed 1.5 times the Interquartile Range (IQR $=3^{\text {rd }}$ Quartile- $1^{\text {st }}$ Quartile).

Figure 2 describes the number of days of stimulation and shows that patients on Protocol 1 had a greater range of values (between 5 and 13 days), with a median of 11 days. The median was 11 days in the two Protocols, but the population was more homogeneous in Protocol 2 (half of the patients had $10-11$ days of stimulation, while in Protocol 1 half of the subjects had 5-11 days of stimulation).
The descriptive statistics findings about the medication dose for each protocol prescribed to women in with an ORPI $<0.5$ in G1 revealed that in Protocol 1, the dose of rFSH alfa and beta varied between 1125 and 3000 International Units (IU), with an average value of $2121.60 \pm 493.896$ IU. The UHMG dosage oscillated between 375 IU (minimum) and 1800 IU (maximum) with an average value of $935.19 \pm 290.737$ IU. The GnRH antagonist dose varied in this group between $0.25 \mathrm{mg}$ and $2 \mathrm{mg}$, with an average value of $1.28 \pm 0.301 \mathrm{mg}$. The patients in the 38 cycles performed on Protocol 2 were prescribed $150 \mu \mathrm{g}$ of rFSH corifollitropin alfa. The doses of uHMG oscillated between 300 IU (minimum) and 3900 IU (maximum), with an average value of $1851.32 \pm 02.937 \mathrm{IU}$. The $\mathrm{GnRH}$ antagonist dose varied between $0.75 \mathrm{mg}$ and $2.25 \mathrm{mg}$, with an average value of $1.520 \pm 0.331 \mathrm{mg}$.

In general, we observed that the average doses of uHMG and GnRH antagonists were higher in Protocol 2 than in Protocol 1. The comparison of the proportion of mature oocytes (mean number of mature oocytes / mean number of oocytes obtained) using different triggers is shown in Tables 1 and 2.

In this group, there was no evidence of a significant difference in the proportion (percentage) of mature oocytes for different triggers ( $p$-value $>0.05)$. The values did not differ significantly, although in the group given GnRH agonists there were approximately $12 \%$ more mature oocytes than in the group given rhCG.

Protocol 1 was prescribed to $93.5 \%(n=43)$ and Protocol 2 to $6.5 \%(n=3)$ of the women with normal ovarian reserve (ORPI [0.5-0.9]) in G2. As for the average number of oocytes obtained, there was no statistical evidence to reject the null hypothesis of equality between the two protocols, $t$ (44) $=0.559, p$-value $=0.579$. The average number of oocytes obtained with Protocol 1 (10.91 44.710 oocytes) and with Protocol 2 (9.33 \pm 4.726 oocytes) did not differ significantly. The number of stimulation days was significantly higher in Protocol 2, t (44) $=-2.130, p$-value $<0.05$, with an average difference of 1.6 days. Patients on Protocol 2 had an average

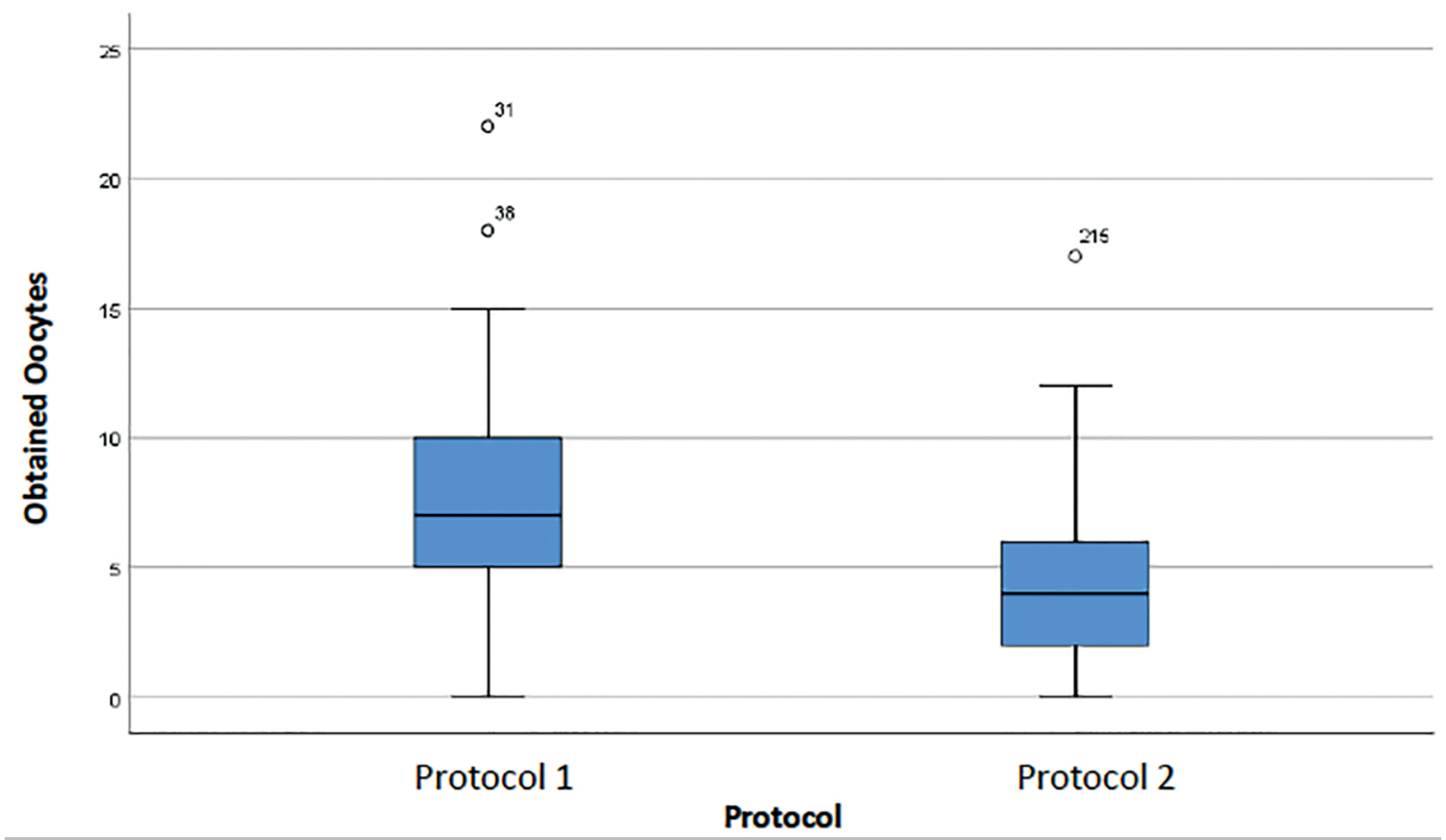

Figure 1. Oocytes retrieved from cycles performed in women with an ORPI $<0.5$ for each protocol. 


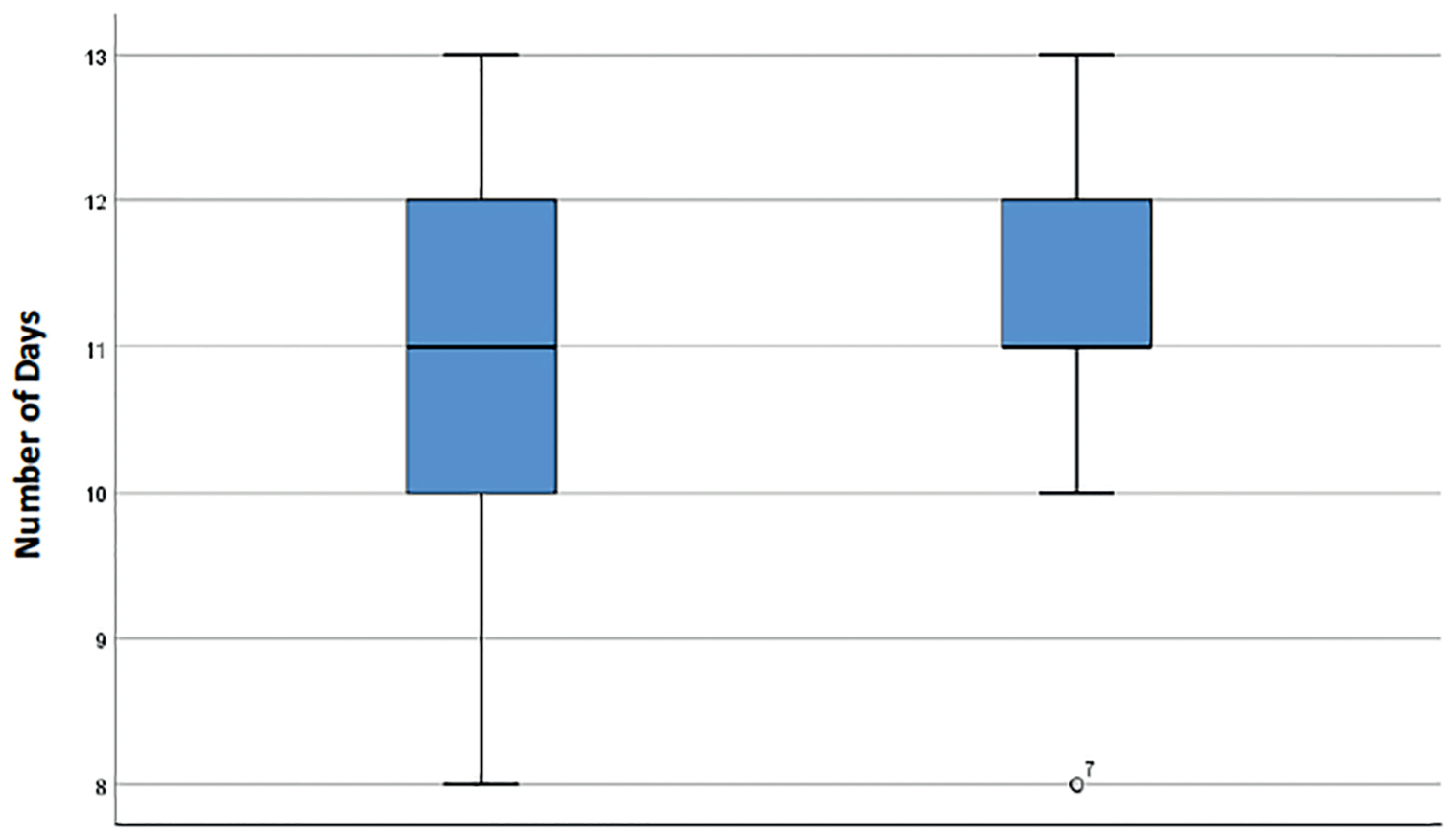

Protocol 1

Protocol 2

Protocol

Figure 2. Number of days of stimulation in cycles of women with an ORPI $<0.5$ for each protocol.

\begin{tabular}{|c|c|c|c|c|c|}
\hline & & Frequency & Percentage & $\begin{array}{c}\text { Valid } \\
\text { Percentage }\end{array}$ & $\begin{array}{l}\text { Cumulative } \\
\text { Percentage }\end{array}$ \\
\hline \multirow{3}{*}{ Valid } & rhCG (Ovitrelle ${ }^{\circledR}$ ) & 113 & 95.0 & 97.4 & 97.4 \\
\hline & GnRH agonist (Decapepty ${ }^{\circledR} /$ Gonapepty ${ }^{\circledR}{ }^{\circledR}$ ) & 3 & 2.5 & 2.6 & 100.0 \\
\hline & Total & 116 & 97.5 & 100.0 & \\
\hline Missing & System & 3 & 2.5 & & \\
\hline Total & 119 & 100.0 & & & \\
\hline
\end{tabular}

\begin{tabular}{|c|c|c|c|c|c|c|c|c|c|}
\hline & & \multirow{2}{*}{$\mathbf{n}$} & \multirow{2}{*}{ Average } & \multirow{2}{*}{$\begin{array}{l}\text { Standard } \\
\text { deviation }\end{array}$} & \multirow{2}{*}{$t$ (df) } & \multirow{2}{*}{$p$-value } & \multirow{2}{*}{$\begin{array}{c}\text { Mean } \\
\text { difference }\end{array}$} & \multicolumn{2}{|c|}{$\begin{array}{l}\text { 95\% Confidence } \\
\text { Interval }\end{array}$} \\
\hline & & & & & & & & $\begin{array}{l}\text { Inferior } \\
\text { limit }\end{array}$ & $\begin{array}{l}\text { Superior } \\
\text { limit }\end{array}$ \\
\hline \multirow{2}{*}{$\begin{array}{l}\text { Mature } \\
\text { oocytes }\end{array}$} & rhCG (Ovitrelle ${ }^{\circledR}$ ) & 113 & 0.7759 & 0.24219 & \multirow{2}{*}{$\begin{array}{l}-0.830 \\
(144)\end{array}$} & \multirow[b]{2}{*}{0.408} & \multirow[b]{2}{*}{-0.117} & \multirow[b]{2}{*}{-0.39558} & \multirow[b]{2}{*}{0.16191} \\
\hline & 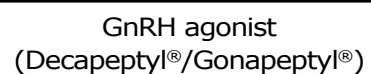 & 3 & 0.8928 & 0.11625 & & & & & \\
\hline
\end{tabular}

of $12 \pm 1.00$ days of stimulation, while subjects in Protocol 1 had an average of $10.37 \pm 1.291$ days of stimulation.

Figure 3 shows the median number of oocytes obtained from the two protocols ( 9 in Protocol 1 and 11 in Protocol 2 ). As for the number of days of stimulation (Figure 4), subjects on Protocol 2 were on stimulation for a median of 12 days ( $50 \%$ of the population were on stimulation for up to 12 days), while patients on Protocol 1 were on stimulation for a median of 10.5 days.
The dose of rFSH alfa and beta prescribed to patients on Protocol 1 ranged from 900 IU and 2700 IU, with an average value of $1777.91 \pm 479.186$ IU. The dose of uHMG oscillated between $375 \mathrm{IU}$ and $1800 \mathrm{IU}$, with an average value of $856.40 \pm 305.850 \mathrm{IU}$. The dose of $\mathrm{GnRH}$ antagonists varied in this group between $0.75 \mathrm{mg}$ and $1.75 \mathrm{mg}$, with an average value of $1.2209 \pm 0.31908 \mathrm{mg}$. The three cycles performed in patients on Protocol 2 used a dose of $150 \mu \mathrm{g}$ of rFSH corifollitropin alfa. The dose of uHMG oscillated between 1800IU 


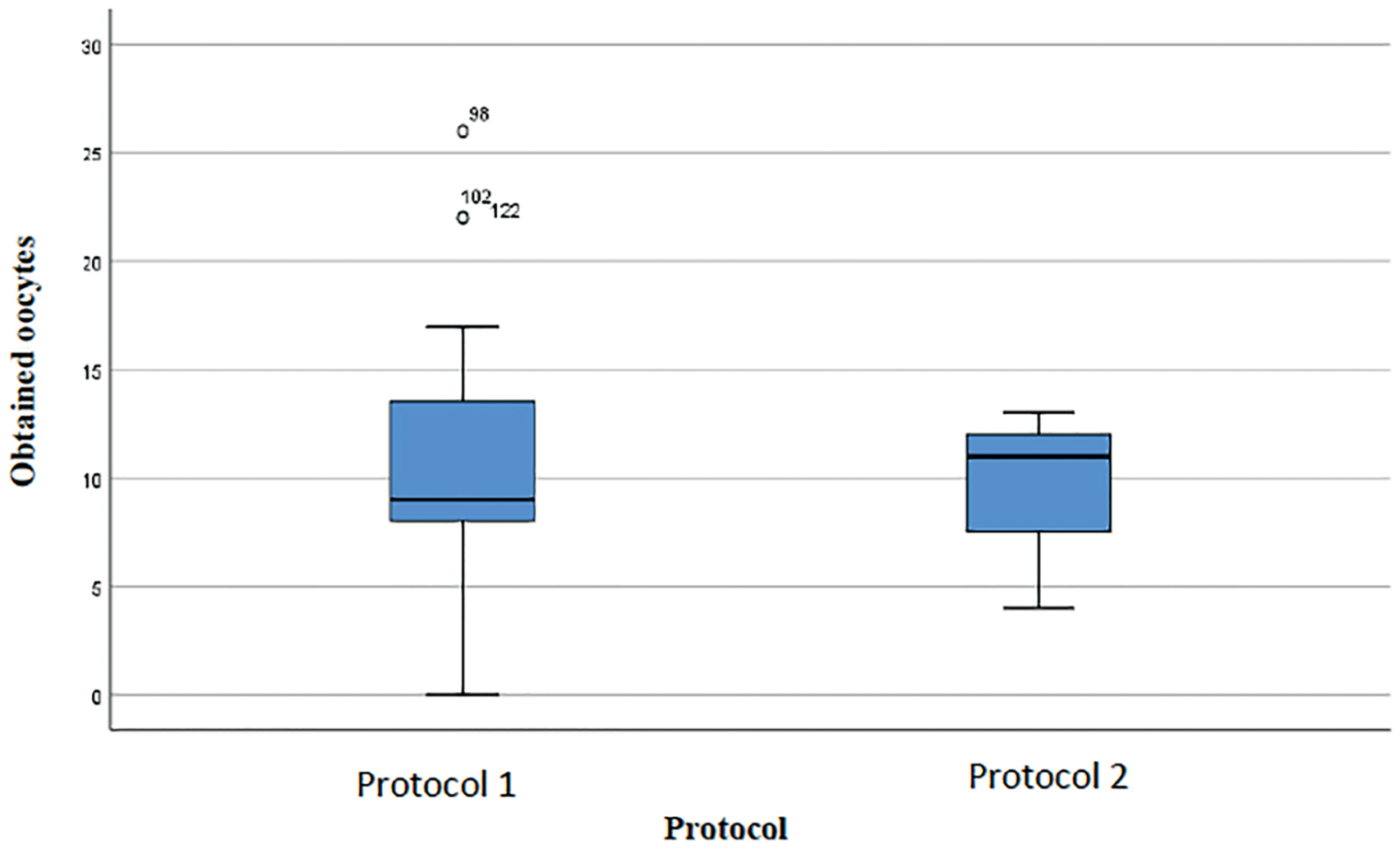

Figure 3. Oocytes retrieved from the cycles of women with an ORPI [0.5-0.9] for each protocol.

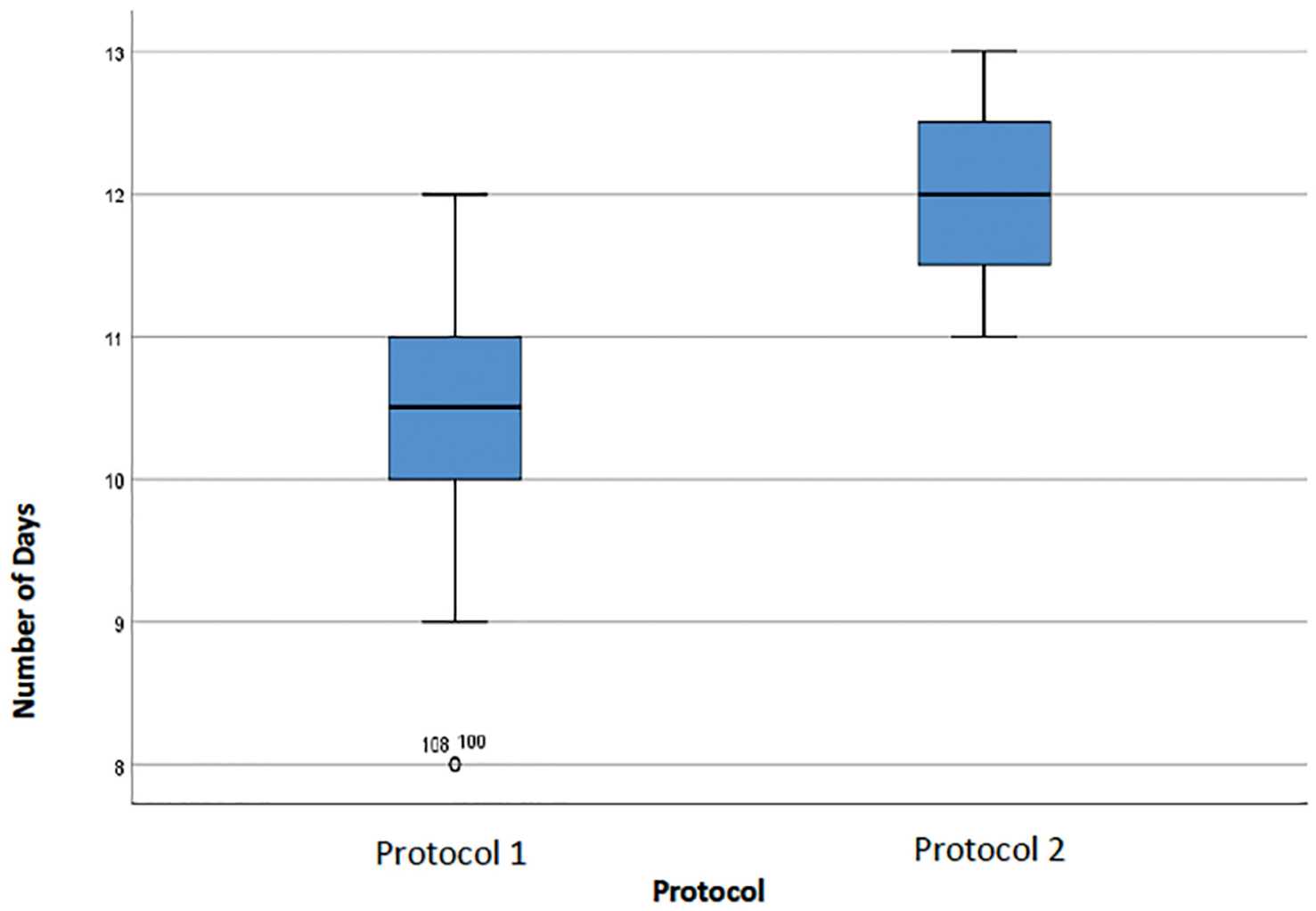

Figure 4. Number of days of stimulation in the cycles of women with an ORPI [0.5-0.9] for each protocol.

and 2700IU, with an average value of $2200 \pm 458.258$ IU. The dose of GnRH antagonists varied between $1 \mathrm{mg}$ and $2 \mathrm{mg}$, with an average value of $1.6667 \pm 0.57735 \mathrm{mg}$. In general, we observed that the average doses of uHMG and GnRH antagonists were higher in Protocol 2 than in Protocol 1. The com- parison of the proportion of mature oocytes (mean number of mature oocytes / mean number of oocytes obtained) using different triggers is shown in Tables 3 and 4.

In this group, there was no statistical evidence to reject the null hypothesis of equality of the percentage of ma- 
Table 3. Classification of the cycles of women with an ORPI of [0.5-0.9] based on the trigger.

\begin{tabular}{|c|c|c|c|c|c|}
\hline & & Frequency & Percentage & Valid Percentage & $\begin{array}{c}\text { Cumulative } \\
\text { Percentage }\end{array}$ \\
\hline \multirow{3}{*}{ Valid } & rhCG $\left(\right.$ Ovitrelle $\left.^{\circledR}\right)$ & 41 & 89.1 & 89.1 & 89.1 \\
\cline { 2 - 6 } & $\begin{array}{c}\text { GnRH agonist } \\
\text { (Decapepty| }{ }^{\circledR} / \text { Gonapepty }{ }^{\circledR} \text { ) }\end{array}$ & 5 & 10.9 & 10.9 & 100.0 \\
\cline { 2 - 6 } & Total & 46 & 100.0 & 100.0 & \\
\hline
\end{tabular}

\begin{tabular}{|c|c|c|c|c|c|c|c|c|c|}
\hline & & \multirow{2}{*}{$\mathbf{n}$} & \multirow{2}{*}{ Average } & \multirow{2}{*}{$\begin{array}{l}\text { Standard } \\
\text { deviation }\end{array}$} & \multirow{2}{*}{$t$ (df) } & \multirow{2}{*}{$p$-value } & \multirow{2}{*}{$\begin{array}{c}\text { Mean } \\
\text { difference }\end{array}$} & \multicolumn{2}{|c|}{$\begin{array}{c}\text { 95\% Confidence } \\
\text { interval }\end{array}$} \\
\hline & & & & & & & & $\begin{array}{l}\text { Inferior } \\
\text { limit }\end{array}$ & $\begin{array}{l}\text { Superior } \\
\text { limit }\end{array}$ \\
\hline \multirow{2}{*}{$\begin{array}{l}\text { Mature } \\
\text { oocytes }\end{array}$} & rhCG (Ovitrelle ${ }^{\circledR}$ ) & 41 & 0.7905 & 0.18711 & \multirow{2}{*}{$\begin{array}{c}-0.119 \\
(44)\end{array}$} & \multirow[b]{2}{*}{0.906} & \multirow[b]{2}{*}{0.001} & \multirow[b]{2}{*}{-0.18562} & \multirow[b]{2}{*}{0.16486} \\
\hline & $\begin{array}{c}\text { GnRH agonist } \\
\left.\text { (Decapepty }{ }^{\circledR} / \text { Gonapeptyl }{ }^{\circledR}\right)\end{array}$ & 5 & 0.8009 & 0.14338 & & & & & \\
\hline
\end{tabular}

ture oocytes with each trigger ( $p$-value $>0.05)$. The values were similar, and the difference, a residual $1 \%$.

Protocol 1 was prescribed to $97.5 \%(n=79)$ and Protocol 2 to $2.5 \%(n=2)$ of the women with high ovarian reserve (ORPI $\geq 0.9$ ) in $\mathrm{G} 3$. In this group, the number of oocytes obtained did not differ significantly between protocols: in Protocol 1, the average was 12.18 16.207 oocytes; in Protocol 2, it was $4.00 \pm 0.00$ oocytes (zero variability, as seen in the boxplot: the two subjects in this condition obtained 4 oocytes), with a $p$-value of 0.068 . Regarding the average number of days of stimulation of the two protocols, there was no statistical evidence to reject the null hypothesis of equality $(p$-value $>0.05)$. The following boxplots represent the distribution of data for each protocol regarding the number of oocytes obtained and the number of stimulation days. In Figure 5, despite the higher median number of obtained oocytes in Protocol 1, we could not infer a trend for the study population ( $p$-value $>0.05$ ).

Although the median length of stimulation was higher in Protocol 2 (Figure 6), the difference in relation to Protocol 1 was not statistically significant. In terms of the dosages for each medication prescribed, patients on Protocol 1 were prescribed between 950 and 2475 IU of rFSH alfa and beta, with an average value of $1411.01 \pm 322.298$ IU. The dose of uHMG oscillated between 225 IU (minimum) and 1425 IU (maximum), with an average value of $652.78 \pm 236.326$ IU. The dose of GnRH antagonists varied in this group between 0.50 $\mathrm{mg}$ and $1.75 \mathrm{mg}$, with an average value of $1.1930 \pm 0.24334$ $\mathrm{mg}$. The dose of $150 \mu \mathrm{g}$ of rFSH corifollitropin alfa was used in the two cycles performed with patients on Protocol 2. The dose of uHMG oscillated between 1800 IU (minimum) and 2100 IU (maximum), with an average value of $1950 \pm 212.132$ IU. The dose of $\mathrm{GnRH}$ antagonists varied between $1.50 \mathrm{mg}$ and $1.75 \mathrm{mg}$, with an average value of $1.6250 \pm 0.17678 \mathrm{mg}$. In general, we observed that the average doses of uHMG and GnRH antagonists were higher in Protocol 2 than in Protocol 1 . The comparison of the proportion of mature oocytes (mean number of mature oocytes / mean number of oocytes obtained) using different triggers is shown in Tables 5 and 6 . In this group, there was no statistical evidence to reject the null hypothesis of equality in the percentage of mature oocytes with the different triggers ( $p$-value $>0.05)$. The values were similar, and the average difference, a residual $2 \%$.

\section{DISCUSSION}

Similarly to reports from other countries, this study found that Portuguese patients seek ART therapies at a more advanced age, as evinced by the mean age of the individuals included in our study population (34.51 years). A significant portion of study participants was described as having low ovarian reserve $(48.4 \%)$. Social, biological, and public health factors contribute to this significant increase in infertility cases. Knowledge about the management of follicular stimulantion agents (UHMG and rFSH) has increased the confidence in the protocols prescribed in this study, resulting in prescription to a greater number of patients (Protocol 1 in $82.5 \%$ and Protocol 2 in $17.5 \%$ ). In the last five years, the advent of a new method to protect patients against spontaneous LH surges coupled with better control of COS has allowed for better pregnancy rates and the possibility of avoiding severe OHSS, one of the most frequent and feared complications of IVF/ICSI procedures. According to the 2020 Guidelines of the European Society of Human Reproduction and Embryology (ESHRE) (Ovarian Stimulation TEGGO et al., 2020), the use of protocols with GnRH antagonists is recommended over the use of protocols with GnRH agonists, given the comparable effectiveness, but greater safety of GnRH antagonists. In both protocols under study, the association of rFSH with uHMG (FSH + LH) was used, due to the possibility of greater sensitization of oocyte FSH receptors when exposed to $\mathrm{LH}$ and circulating endogenous estrogen. However, there is still controversy regarding this association. Some studies reported no benefit in combining rFSH with uHMG, compared with rFSH alone, in protocols in which $\mathrm{GnRH}$ antagonists were used (Martin-Johnston et al., 2007). Other studies reported that the association led to an increase in the proportion of oocytes obtained, when compared with the use of rFSH or uHMG alone (Pacchiarotti et al., 2016).

Other studies have reported that patients on pituitary suppression induced by GnRH analogs (either agonists or antagonists) may experience profound LH suppression, resulting in lower fertilization rates and increased risk of miscarriage. These patients require high doses of FSH and have better implantation rates with the addition of $\mathrm{LH}$ to the FSH stimulation protocol (Fleming et al., 1998). 


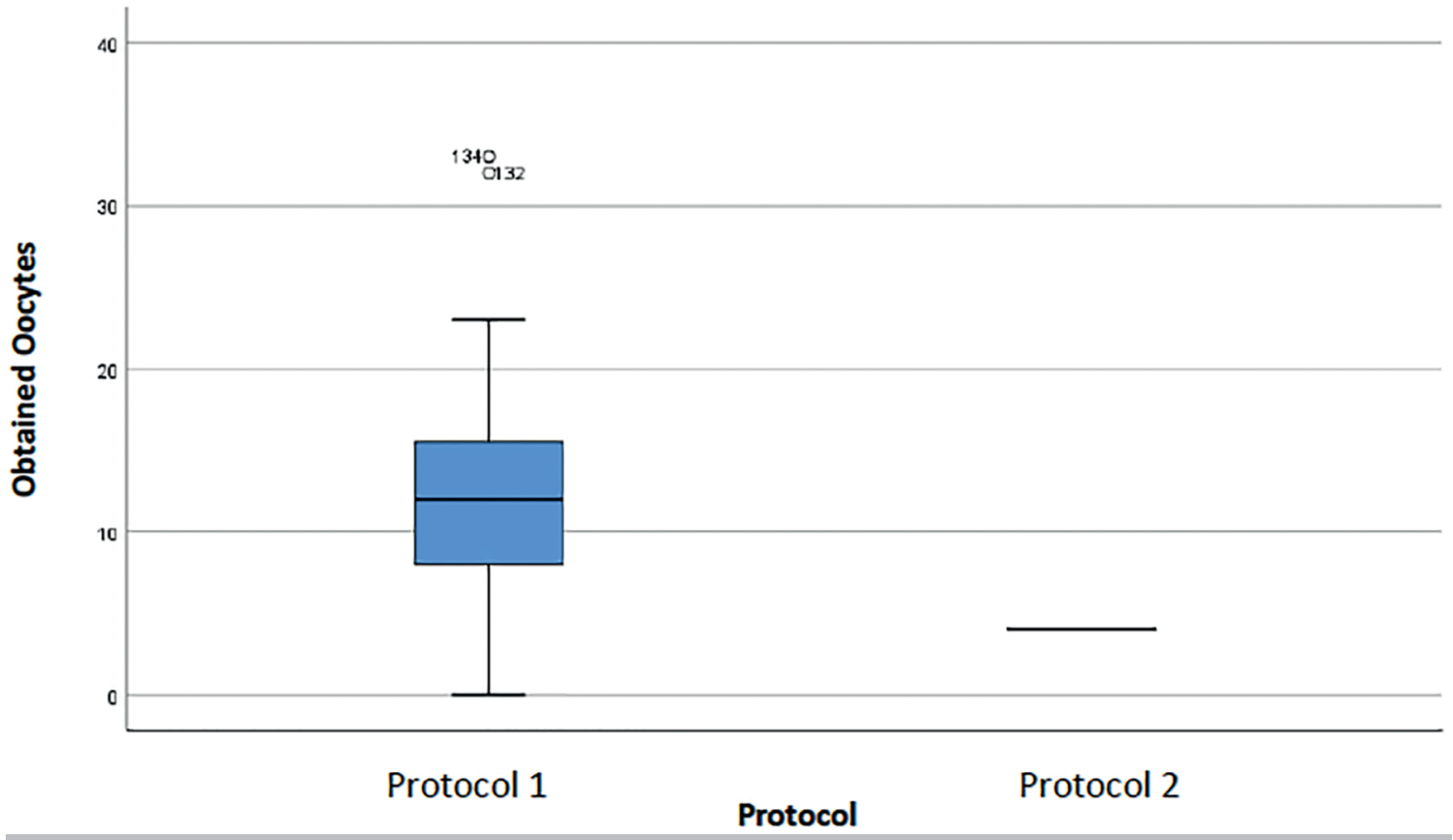

Figure 5. Oocytes retrieved from the cycles of women with an ORPI $\geq 0.9$ for each protocol.

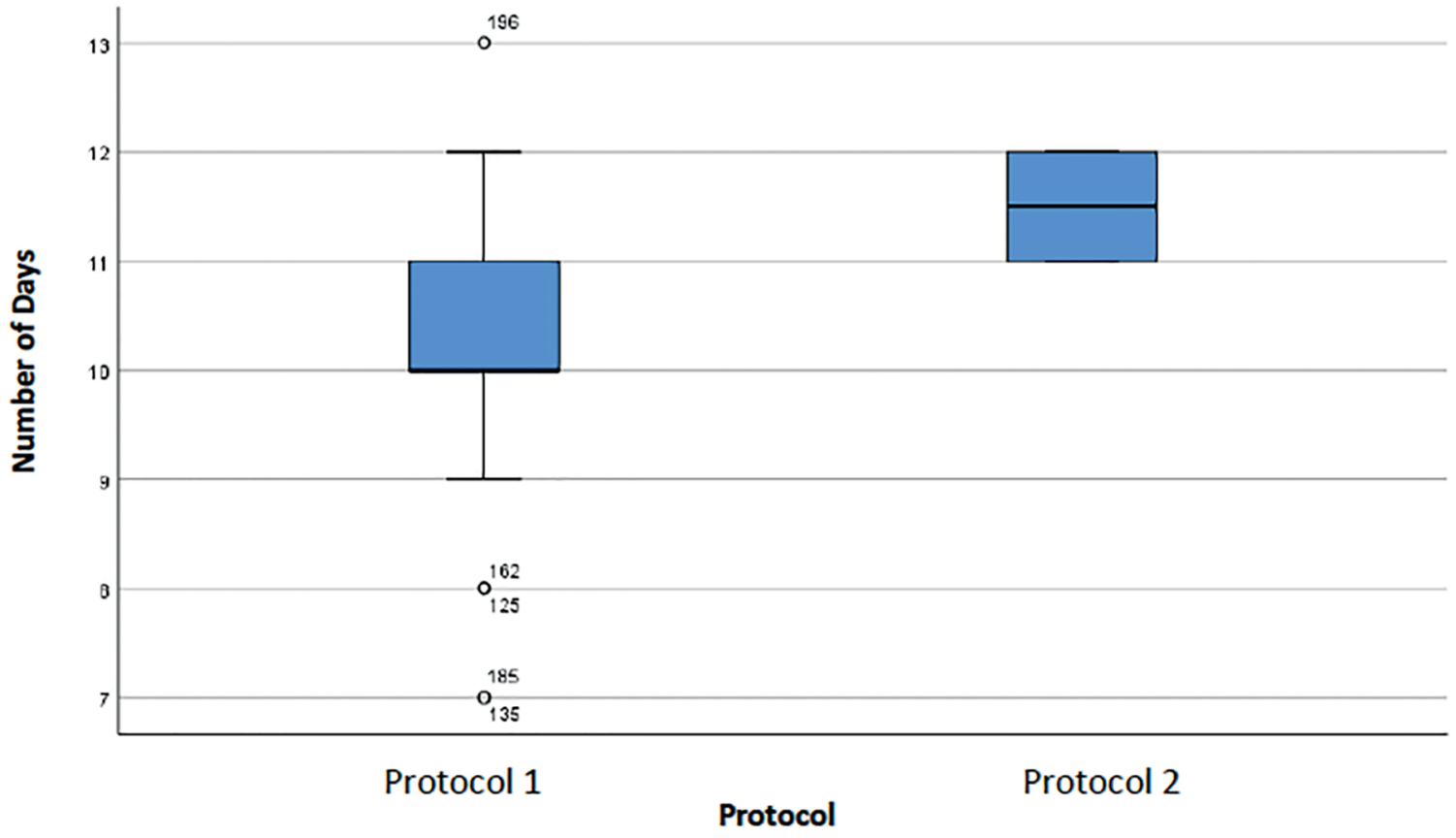

Figure 6. Number of days of stimulation in the cycles of women with an ORPI $\geq 0.9$ for each protocol.

Table 5. Classification of the cycles of women with an ORPI $\geq 0.9$ based on the trigger.

\begin{tabular}{|c|c|c|c|c|c|}
\hline & & Frequency & Percentage & Valid Percentage & $\begin{array}{c}\text { Cumulative } \\
\text { Percentagem }\end{array}$ \\
\hline \multirow{3}{*}{ Valid } & rhCG $\left(\right.$ Ovitrelle $\left.^{\circledR}\right)$ & 62 & 76.5 & 76.5 & 76.5 \\
\cline { 2 - 6 } & $\begin{array}{c}\text { GnRH agonist } \\
\text { (Decapepty }{ }^{\circledR} / \text { Gonapepty }{ }^{\circledR} \text { ) }\end{array}$ & 19 & 23.5 & 23.5 & 100.0 \\
\cline { 2 - 7 } & Total & 81 & 100.0 & 100.0 & \\
\hline
\end{tabular}




\begin{tabular}{l}
\hline $\begin{array}{l}\text { Table 6. Student's t-test comparing the proportion of mature oocytes with the trigger, in the cycles of women with an } \\
\text { ORPI } \geq 0.9 .\end{array}$. \\
\hline
\end{tabular}

The difference between the protocols under analysis lies in the way the prescribed gonadotropins were used. In Protocol 1, rFSH alfa or beta was used in daily doses; in Protocol 2, a deposit form with prolonged release rFSH (corifollitropin alfa) was used. The maximum daily dose of rFSH alfa or beta has been widely studied and should not exceed 300 IU daily. The total dose used to be defined based on the number of days required to reach oocyte maturation. Regarding corifollitropin alfa, even though the maximum dose is inversely proportional to body weight $(100 \mu \mathrm{g}$ in women $<60 \mathrm{~kg}$ and $150 \mu \mathrm{g}$ in women $>60 \mathrm{~kg}$ ) to protect against OHSS, in patients with low reserve or poor response to prior COS ( $<6$ oocytes), the maximum dose $(150 \mu \mathrm{g})$ is recommended in order to produce better ovarian response, regardless of body weight.

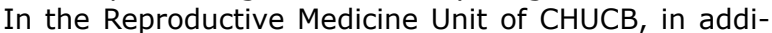
tion to the pre-determined COS protocols, COCs are also used to schedule and date patient menstruation, so as to facilitate scheduling of cycles and minimize the operational costs of IVF/ICSI procedures by optimizing the use of growth medium, equipment, and working time. According to studies (Biljan et al., 1998; Griesinger et al., 2008), the use of COC offers other advantages, such as improving follicular synchronism during ovulation stimulation, decreasing cancellation rates in hyper-responsive women, and yielding a greater number of oocytes. However, according to the ESHRE guidelines (Ovarian Stimulation TEGGO et al., 2020) mentioned above, the use of COC before COS is not recommended in protocols in which $\mathrm{GnRH}$ antagonists are used since they may decrease treatment efficacy, as evidence indicates that pregnancy rates are lower pre-treatment with $\mathrm{COC}$ is performed. However, it is difficult to assess the interference of pre-treatment with COC on outcomes of full-term pregnancy, since in a COS cycle there are numerous steps that may interfere with the final outcome. Regarding the number of oocytes retrieved from OPU, numbers were higher when recombinant follitropin alfa and beta were used in the three study groups compared with deposited corifollitropin alfa.

This difference was significant $(p$-value $<0.001)$ only in the group of women with a low ovarian reserve of an ORPI <0.5. Other studies have found different results. A clinical trial published in 2015 (Kolibianakis et al., 2015) showed that, in a certain group of women with a predictable weak response to COS, there was no significant difference between the use of the different types of rFSH. The ESHRE guidelines (Ovarian Stimulation TEGGO et al., 2020) state that the use of daily rFSH or long-acting rFSH is also recommended in cycles in which GnRH antagonists are used in women expected to have good response to COS. Further studies are certainly needed. The length of stimulation required compared with the ten-day pattern of predicted follicular evolution was significantly higher ( $p$-value $<0.05)$ with the use of corifollitropine alfa in two of the groups ( $\mathrm{G} 1$ and $\mathrm{G} 2$ ). Length of stimulation was added by one day in the low reserve group and two days in the normal reserve group. The need for complementation with HMG in greater quantities is inherent to this protocol. Regarding the number of hormones administered necessary for COS, it is possible to verify that the total dose of rFSH alfa or beta / urinary HMG used was inversely proportional to the patient's ovarian reserve. In other words, the lower the ORPI value, the higher the average number of IU administered (Oliani et al., 2019; Oliveira et al., 2012). Thus, it is possible to understand the importance of knowing the potential ovarian response of a woman in the individualization of the medication dose, so as to try and avoid either a weak response to treatment or hyperstimulation, which may lead to OHSS.

We could also verify that there was a need for complementation with uHMG in higher doses when corifollitropin alfa was used as an rFSH agent. The dosage of the GnRH antagonist is dependent on the number of COS days until the trigger is used, since the antagonist's goal is to prevent a premature LH surge and thus prevent the loss of the cycle resulting from early ovulation. The higher mean dose of antagonists required with the use of corifollitropin alfa relates to the greater number of days of stimulation required with this protocol. In both protocols, daily doses of $0.25 \mathrm{mg}$ were used, according to a flexible protocol (in which the antagonist is introduced when at least one follicle with> $14 \mathrm{~mm}$ is seen in the control ultrasound) (Tarlatzis et al., 2006; Al-Inany et al., 2007). Regarding the trigger used, there was no significant difference between the use of rhCG or GnRH agonists in obtaining mature oocytes. The percentages of mature oocytes were similar in the three ORPI groups and varied between $78 \%$ and $89 \%$ in terms of effectiveness, regardless of the trigger used. The ESHRE guideline (Ovarian Stimulation TEGGO et al., 2020) also shows that, in women with predictable poor response to treatment (poor responders and low reserve) and predictable good response (normal reserve), only uhCG or rhCG should be used as a trigger, while GnRH agonists should be avoided. The latter should be reserved for the final maturation of oocytes in women at risk of hyperstimulation (increased reserve), since it has been associated with a lower risk of OHSS compared with hCG.

The patients in this study prescribed GnRH agonists to trigger ovulation did not have embryos transferred, since they were cryopreserved for embryo transfer (CET) in a natural cycle. With GnRH agonists, the total amount of gonadotropins released to support the formation of the corpus luteum is not adequate, which thus leads to premature luteolysis and poor reproductive results (Yilmaz et al., 2020). Thus, the use of a GnRH agonist for final maturation and fresh transfer is not recommended in the general IVF / ICSI population (Ovarian Stimulation TEGGO et al., 2020). With the increase in the effectiveness of cryopreservation, the use of GnRH agonists with a "freeze all" approach so that embryos are transferred in subsequent cycles may be the ideal strategy to decrease or eliminate the prevalence of OHSS (YIlmaz et al., 2020). The main limitations of this 
study are the sample size and the number of COS protocols compared. In the future, we believe this study should be extended to cover a larger population, compare between other COS protocols, and use data from other Reproductive Medicine Units in the country, so that a more thorough analysis is performed.

\section{CONCLUSION}

From the available data, it is possible to conclude that follitropin alfa or beta (Protocol 1 ) yielded a greater number of oocytes retrieved in OPU than corifollitropin alfa (Protocol 2) for all ORPI ranges, with a significant difference $(p<0.001)$ seen in the low reserve group (G1); the dose of rFSH alfa or beta used in conjunction with uHMG (Protocol 1) increases in an inversely proportional manner to the ORPI value; the fixed dose of rFSH deposit used in Protocol 2 requires a sharp increase in the dose of uHMG, also reflected in the number of COS days and, consequently, in the number of ampoules used by the GnRH antagonist; and the final maturation (trigger) to obtain mature oocytes (MII) has the same efficiency with the use of rhCG or a GnRH agonist. Further studies, using ORPI as an additional input for the selection of users directed to ART procedures, are necessary for the implementation of other cos protocols.

\section{ACKNOWLEDGEMENTS}

We would like to thank the staff from the Reproductive Medicine Unit - University Hospital Center Cova da Beira Portugal - involved in this research.

\section{CONFLICT OF INTEREST}

All authors declare that there was no support from any organization for the submitted paper, no financial relationships with any organizations that might have an interest in the submitted study and no other relationships or activities that might appear to have influenced the submitted study.

\section{Corresponding author:}

Antonio Helio Oliani

Reproductive Medicine Unit

University Hospital Center

Cova da Beira - Portugal

Institute of Reproductive and Fetal Medicine

Faculty of Medicine of São José do Rio Preto - Brazil

E-mail: aholiani@gmail.com

\section{REFERENCES}

Al-Inany HG, Abou-Setta AM, Aboulghar M. Gonadotrophin-releasing hormone antagonists for assisted conception: a Cochrane review. Reprod Biomed Online. 2007;14:640-9. PMID: 17509210 DOI: 10.1016/S14726483(10)61059-0

Alyasin A, Mehdinejadiani S, Ghasemi M. GnRH agonist trigger versus hCG trigger in GnRH antagonist in IVF/ICSI cycles: A review article. Int J Reprod Biomed. 2016;14:55766. PMID: 27738657 DOI: 10.29252/ijrm.14.9.557

Amanvermez R, Tosun M. An Update on Ovarian Aging and Ovarian Reserve Tests. Fertil Steril. 2016;9:411-5. PMID: 26985328 DOI: $10.22074 /$ ijfs.2015.4591

Biljan MM, Mahutte NG, Dean N, Hemmings R, Bissonnette $F$, Tan SL. Effects of pretreatment with an oral contraceptive on the time required to achieve pituitary suppression with gonadotropin-releasing hormone analogues and on subsequent implantation and pregnancy rates. Fertil Steril. 1998;70:1063-9. PMID: 9848296 DOI: 10.1016/S00150282(98)00333-1
Blumenfeld Z. The Ovarian Hyperstimulation Syndrome. Vitam Horm. 2018;107:423-51. PMID: 29544639 DOI: 10.1016/bs.vh.2018.01.018

Braga DPAF, Zanetti BF, Setti AS, Iaconelli A Jr, Borges E Jr. Immature oocyte incidence: Contributing factors and effects on mature sibling oocytes in intracytoplasmic sperm injection cycles. JBRA Assist Reprod. 2020;24:70-6. DOI: 10.5935/1518-0557.20190056 PMID: 31589389

Deeks ED. Highly Purified Human Menopausal Gonadotropin (Menopur $®$ ): A Profile of Its Use in Infertility. Clin Drug Investig. 2018;38:1077-84. PMID: 30264288 DOI: $10.1007 / \mathrm{s} 40261-018-0703-8$

Deshpande PS, Gupta AS. Causes and Prevalence of Factors Causing Infertility in a Public Health Facility. J Hum Reprod Sci. 2019;12:287-93. PMID: 32038077 DOI: 10.4103/jhrs.JHRS_140_18

Dewailly D, Laven J. AMH as the primary marker for fertility. Eur J Endocrinol. 2019;181:D45-51. PMID: 31398713 DOI: $10.1530 / E J E-19-0373$

Direcção Geral de Saúde. Saúde Reprodutiva: Infertilidade Cuidados de Saúde Hospitalares. Lisboa: Direcção Geral de Saúde; 2010. Available at: https://www.arscentro.min-saude. pt/Institucional/projectos/crsmca/noc/Documents/saude $\% 20$ materna/2\%20Conduta\%20em\%20Infertilidade\%202011. pdf

Dosouto C, Haahr T, Humaidan P. Gonadotropin-releasing hormone agonist (GnRHa) trigger - State of the art. Reprod Biol. 2017;17:1-8. PMID: 28215488 DOI: 10.1016/j. repbio.2017.01.004

Fleming R, Lloyd F, Herbert M, Fenwick J, Griffiths T, Murdoch A. Effects of profound suppression of luteinizing hormone during ovarian stimulation on follicular activity, oocyte and embryo function in cycles stimulated with purified follicle stimulating hormone. Hum Reprod. 1998;13:178892. PMID: 9740424 DOI: 10.1093/humrep/13.7.1788

Gnoth C, Godehardt E, Frank-Herrmann P, Friol K, Tigges J, Freundl G. Definition and prevalence of subfertility and infertility. Hum Reprod. 2005;20:1144-7. PMID: 15802321 DOI: $10.1093 /$ humrep/deh870

Griesinger G, Venetis CA, Marx T, Diedrich K, Tarlatzis BC, Kolibianakis EM. Oral contraceptive pill pretreatment in ovarian stimulation with GnRH antagonists for IVF: a systematic review and meta-analysis. Fertil Steril. 2008;90:1055-63. PMID: 18054003 DOI: 10.1016/j.fertnstert.2007.07.1354

Hodgen GD. Biological basis of follicle growth. Hum Reprod. 1989;4:37-46. PMID: 2693488 DOI: 10.1093/humrep/4.suppl_1.37

Kolibianakis EM, Venetis CA, Bosdou JK, Zepiridis L, Chatzimeletiou K, Makedos A, Masouridou S, Triantafillidis S, Mitsoli A, Tarlatzis BC. Corifollitropin alfa compared with follitropin beta in poor responders undergoing ICSI: a randomized controlled trial. Hum Reprod. 2015;30:432-40. PMID: 25492411 DOI: 10.1093/humrep/deu301

La Marca A, Sighinolfi G, Radi D, Argento C, Baraldi E, Artenisio AC, Stabile G, Volpe A. Anti-Mullerian hormone $(\mathrm{AMH})$ as a predictive marker in assisted reproductive technology (ART). Hum Reprod Update. 2010;16:113-30. PMID: 19793843 DOI: 10.1093/humupd/dmp036 
Le MT, Nguyen DN, Zolton J, Nguyen VQH, Truong QV, Cao NT, Decherney A, Hill MJ. GnRH Agonist versus hCG Trigger in Ovulation Induction with Intrauterine Insemination: A Randomized Controlled Trial. Int J Endocrinol. 2019;2019:2487067. PMID: 31001335 DOI: 10.1155/2019/2487067

Ledger WL, Fauser BC, Devroey P, Zandvliet AS, Mannaerts BM. Corifollitropin alfa doses based on body weight: clinical overview of drug exposure and ovarian response. Reprod Biomed Online. 2011;23:150-9. PMID: 21665541 DOI: $10.1016 /$ j.rbmo.2011.04.002

Liu K, Case A; Reproductive Endocrinology and Infertility Committee. Advanced reproductive age and fertility. J Obstet Gynaecol Can. 2011;33:1165-75. PMID: 22082792 DOI : $10.1016 / S 1701-2163(16) 35087-3$

Martin-Johnston M, Beltsos AN, Grotjan HE, Uhler ML. Adding human menopausal gonadotrophin to antagonist protocols is there a benefit? Reprod Biomed Online. 2007;15:161-8. PMID: 17697491 DOI: $10.1016 / S 1472-6483(10) 60704-3$

Newton CL, Riekert C, Millar RP. Gonadotropin-releasing hormone analog therapeutics. Minerva Ginecol. 2018;70:497-515. PMID: 30264955 DOI: 10.23736/S0026-4784.18.04316-2

Oliani AH, Dias CM, Vaz-Oliani DCM, Amar MPF, Spressão-Alvares $M$. Monitorização ultrassonográfica da indução da ovulação em alta complexidade. In: Caetano JPJ, Marinho RM, Petracco A, Lopes JRC, Ferriani RA, eds. Medicina Reprodutiva SBRH. São Paulo: Segmento Farma Editores; 2019. p. 185-96.

Oliveira JB, Baruffi RL, Petersen CG, Mauri AL, Nascimento AM, Vagnini L, Ricci J, Cavagna M, Franco JG Jr. A new ovarian response prediction index (ORPI): implications for individualized controlled ovarian stimulation. Reprod Biol Endocrinol. 2012;10:94. PMID: 23171004 DOI: 10.1186/1477-7827-10-94

Ovarian Stimulation TEGGO, Bosch E, Broer S, Griesinger G, Grynberg M, Humaidan P, Kolibianakis E, Kunicki M, La Marca A, Lainas G, Le Clef N, Massin N, Mastenbroek S, Polyzos $N$, Sunkara SK, Timeva T, Töyli M, Urbancsek J, Vermeulen $\mathrm{N}$, Broekmans F. ESHRE guideline: ovarian stimulation for IVF/ICSIt. Hum Reprod Open. 2020;2020:hoaa009. PMID: 32395637 DOI: 10.1093/hropen/hoaa009
Pacchiarotti A, Selman H, Valeri C, Napoletano S, Sbracia M, Antonini G, Biagiotti G, Pacchiarotti A. Ovarian Stimulation Protocol in IVF: An Up-to-Date Review of the Literature. Curr Pharm Biotechnol. 2016;17:303-15. PMID: 26775651 DOI: $10.2174 / 1389201017666160118103147$

Quaas AM, Legro RS. Pharmacology of medications used for ovarian stimulation. Best Pract Res Clin Endocrinol Metab. 2019;33:21-33. PMID: 30470497 DOI: 10.1016/j. beem.2018.10.002

Rombauts L, Talmor A. Corifollitropin alfa for female infertility. Expert Opin Biol Ther. 2012;12:107-12. PMID: 22129170 DOI: $10.1517 / 14712598.2012 .641530$

Silva ALB, Vilodre LCF. Avaliação da reserva ovariana: métodos atuais. Femina. 2009;37:149-54.

Tarlatzis BC, Fauser BC, Kolibianakis EM, Diedrich K, Rombauts L, Devroey P. GnRH antagonists in ovarian stimulation for IVF. Hum Reprod Update. 2006;12:333-40. PMID: 16567347 DOI: $10.1093 /$ humupd/dml001

Usta T, Oral E. Is the measurement of anti-Müllerian hormone essential? Curr Opin Obstet Gynecol. 2012;24:151-7. PMID: 22487725 DOI: $10.1097 / G C O .0 b 013 e 3283527 d c f$

Vander Borght M, Wyns C. Fertility and infertility: Definition and epidemiology. Clin Biochem. 2018;62:2-10. PMID: 29555319 DOI: $10.1016 /$ j.clinbiochem.2018.03.012

Vollenhoven B, Hunt S. Ovarian ageing, and the impact on female fertility. F1000Res. 2018;7:F1000 Faculty Rev-1835. PMID: 30542611 DOI: 10.12688/f1000research.16509.1

Youssef MA, Abou-Setta AM, Lam WS. Recombinant versus urinary human chorionic gonadotrophin for final oocyte maturation triggering in IVF and ICSI cycles. Cochrane Database Syst Rev. 2016;4:CD003719. PMID: 27106604 DOI: 10.1002/14651858.CD003719.pub4

Yılmaz N, Ceran MU, Ugurlu EN, Gülerman HC, Engin Ustun Y. GnRH agonist versus HCG triggering in different IVF/ ICSI cycles of same patients: a retrospective study. J Obstet Gynaecol. 2020;40:837-42. PMID: 31791167 DOI: 10.1080/01443615.2019.1674262 